\title{
Impacto del Covid-19 en las relaciones laborales en Ecuador
}

\author{
Impact of Covid-19 on labor relations \\ in Ecuador
}

\author{
Graciela Monesterolo Lencioni \\ Profesora titular principal PUCE \\ Correo: GAMONESTEROLO@puce.edu.ec \\ Ciudad: Quito \\ País: Ecuador
}

Artículo original (investigación)

RFJ, No 8,Vol. I, 2020, pp. 80-119, ISSN 2588-0837

RESUMEN: Desde el 11-III-20, en que declaró el estado de emergencia sanitaria en el Ecuador por la inminente posibilidad del efecto provocado por el coronavirus Covid-19, se desató una crisis generalizada en todos los órdenes de la vida social. El sector más afectado, sin duda ha sido el de la salud, con la pérdida de miles de personas. No obstante, el ámbito de las relaciones laborales también ha sido fuertemente impactado. Muchas personas trabajadoras han visto alterados sus derechos, tan caramente conquistados. Se han modificado condiciones del trabajo, y lo peor de todo, se han perdido infinitas fuentes de trabajo, privando así a un considerable porcentaje de la población del derecho fundamental al trabajo, con el cual llevar el alimento a sus hogares. La privación o reducción de los ingresos derivados de una relación laboral, repercute a su vez en otros derechos fundamentales, como la alimentación, la vestimenta, la educación, la salud, el ocio. Por ello, este artículo se dedica a las regulaciones emergentes que se expidieron para enfrentar la crisis provocada por el Covid-19 en el Ecuador y de cómo ellas, en muchos casos, inobservaron principios fundamentales del Derecho del Trabajo; aunque ello pudiera considerarse razonable y legítimo dada la situación de extrema excepcionalidad. El análisis se circunscribe a las relaciones laborales de los empleados privados y los obreros, es decir, a los 
trabajadores sujetos al Código del Trabajo. También se refiere, finalmente, a los retos que se presentan para la reactivación económica en los nuevos escenarios y condiciones laborales en lo que se ha denominado "la nueva normalidad", así como al gran desafío que tiene el Derecho del Trabajo para redefinirse, sin perder su razón de ser, y así adaptarse a las exigencias del trabajo del futuro y del futuro del trabajo, como resultado de la globalización, de los avances de la ciencia y de la tecnología, y la consecuente automatización. En definitiva, se refiere a la necesidad de que el nuevo Derecho del Trabajo se proyecte al futuro del mundo del trabajo con una mirada diferente, innovadora y progresista para ser más justo, equitativo e inclusivo.

PALABRAS CLAVES: jornadas de trabajo; principios del Derecho Laboral; regulaciones emergentes.

SUMMARY: Since March 11, 1920, when a state of health emergency was declared in Ecuador due to the imminent possibility of the effect of the coronavirus Covid-19, a generalized crisis has been unleashed in all areas of social life. The most affected sector has undoubtedly been the health sector, with the loss of thousands of people. However, the area of labour relations has also been strongly impacted. Many workers have seen their rights altered, so dearly won. Working conditions have changed, and worst of all, infinite sources of work have been lost, thus depriving a considerable percentage of the population of the fundamental right to work, with which to take food home. The deprivation or reduction of income derived from an employment relationship, in turn, affects other fundamental rights, such as food, clothing, education, health and leisure. Furthermore, this research article is dedicated to the emerging regulations that were issued to confront the crisis provoked by Covid-19 in Ecuador and how they, in many cases, failed to observe fundamental principles of labour law. However, this could be considered reasonable and legitimate, given the situation of extreme exceptionality. The analysis is limited to the labour relations of private employees and workers, i.e., workers subject to the Labour Code. It refers, finally, to the challenges presented by the economic revival in the new 
scenarios and working conditions in what has been called "the new normality", also by the great challenge that Labour Law has to redefine itself, without losing its raison d'être. Thus, for adapting to the demands of future work and the future of labour (as a result of globalisation, advances in science and technology, and the consequent automatization). In short, it refers to the need for the new Labour Law to project itself into the future of the world of work with a different, innovative and progressive outlook in order to be more just, equitable and inclusive.

KEY WORDS: working days; principles of Labour Law; emerging regulations.

\section{UNA NECESARIA CONTEXTUALIZACIÓN. DECLARATORIA DE EMERGENCIA SANITARIA POR COVID-19}

Mediante Acuerdo 126-2020, de 11-III-20, el Ministerio de Salud Pública del Ecuador declaró el estado de emergencia sanitaria por la inminente posibilidad del efecto provocado por el coronavirus COVID-19 (en adelante simplemente COVID-19) y prevenir así un posible contagio masivo en la población. El 16 de marzo el presidente de la República, mediante Decreto Ejecutivo 1017 declaró el estado de excepción por calamidad pública en todo el territorio nacional, por los casos de coronavirus confirmados y la declaratoria de pandemia de COVID-19 por parte de la Organización Mundial de la Salud.

En el mismo Decreto, se declaró toque de queda, es decir, la restricción general de circulación de personas y vehículos en vías y espacios públicos. Se exceptuó del toque de queda a ciertas personas, tales como las que prestan servicios básicos, miembros de la Policía y Fuerzas Armadas, comunicadores sociales acreditados, personas que por razones de salud deban trasladarse a un centro médico y a personas que requieran circular para abastecerse de productos de primera necesidad, entre otras. Adicionalmente, se suspendió la jornada presencial de trabajo para todos los trabajadores y empleados del sector público y privado. Se exceptuaron de esta regla general a quienes prestan servicios públicos básicos, de salud, seguridad, bomberos, riesgos, aeropuertos, terminales aéreos, 
terrestres, marítimos, fluviales, bancarios, provisión de víveres, sectores estratégicos y otros servicios que ayudan a combatir la propagación del COVID-19; estableciéndose que dichas personas podían mantener la jornada laboral presencial. La suspensión de la jornada presencial de trabajo en un primer momento fue solo para los días comprendidos entre el 17 y 24 de marzo de 2020. Sin embargo, esto se fue prolongando y luego flexibilizando, en función de las disposiciones del Comité de Operaciones de Emergencia (COE). Se dispuso también que las industrias, cadenas y actividades comerciales en las áreas de alimentación, salud, cadena de exportaciones, industria agrícola, ganadera y de cuidado de animales siguieran funcionando. Expresamente se ordenó que los supermercados, tiendas, bodegas y centros de expendio de víveres y medicinas no cierren sus puertas, y que las plataformas digitales de entregas a domicilios continúen con sus servicios, para así garantizar los servicios básicos de alimentación y salud.

Es así como desde entonces se desató una grave crisis en los diferentes órdenes de la vida social. El ámbito de la salud ha sido el más afectado, y se lamentan hasta el momento alrededor de 600.000 muertes a nivel mundial, cifras que lamentable e inevitablemente seguirán aumentando. Si bien nada puede compararse con la pérdida del más preciado de los derechos humanos, la vida, no se puede desconocer que la pandemia también ha impactado grandemente a la economía de los 218 países en los que se ha dado el brote del virus mortal. $\mathrm{Y}$, como siempre, quienes se encuentran en situación de mayor vulnerabilidad son los que reciben la peor parte.

El ámbito de las relaciones laborales también ha sido fuertemente afectado. Muchas personas trabajadoras han visto alterados sus derechos, tan caramente conquistados. Se han modificado condiciones del trabajo, y lo peor de todo, se han perdido infinitas fuentes de trabajo, privando así a un considerable porcentaje de la población del derecho fundamental al trabajo, con el cual llevar el alimento a sus hogares. La falta de un trabajo o la reducción de las horas laborables, con la consecuente privación o reducción de los ingresos, respectivamente, repercute a su vez en otros derechos fundamentales como la alimentación, la vestimenta, la educación, la salud, el ocio. 
Por todo lo dicho, dedicaremos este artículo a analizar las regulaciones emergentes que se expidieron para enfrentar la crisis provocada por el COVID-19 en el Ecuador, y de cómo ellas, en muchos casos, inobservaron principios fundamentales del Derecho del Trabajo. También nos referiremos a los retos que se presentan para la reactivación económica en los nuevos escenarios y condiciones laborales en lo que se ha denominado "la nueva normalidad". Dicho análisis se limitará a las relaciones laborales en los empleados privados y los obreros, es decir, los trabajadores sujetos al Código del Trabajo. También nos al gran desafío que tiene el Derecho del Trabajo para redefinirse, sin perder su razón de ser, y así adaptarse a las exigencias del trabajo del futuro y del futuro del trabajo, como resultado de la globalización, de los avances de la ciencia y de la tecnología, y la consecuente automatización. En definitiva, nos referiremos a la necesidad de que el nuevo Derecho del Trabajo se proyecte al futuro del mundo del trabajo con una mirada diferente, innovadora y progresista para ser más justo, equitativo e inclusivo.

\section{REPERCUSIONES DE LA DECLARATORIA DE EMERGENCIA SANITARIA EN LOS SECTORES PRODUCTIVOS Y LABORALES}

En el Ecuador, el tejido empresarial está compuesto por aproximadamente un millón de unidades productivas que generan más de tres millones de plazas de empleo. Dentro de este tejido, las micro, pequeñas y medianas empresas representan el 99\%, generando el 68\% del empleo. En virtud de la emergencia sanitaria, las unidades productivas tuvieron que paralizar sus actividades, constituyéndose en unos de los segmentos más afectados debido a la falta de ingresos, falta de liquidez, reducción de la demanda (nacional e internacional), dificultad para acceder a insumos, reducción o falta de mano de obra, difícil acceso a financiamiento y procesos productivos que no están ajustados para ser operados de manera remota o automatizada, lo que podría aumentar el cierre de muchas unidades productivas y a su vez, la desvinculación de muchos trabajadores y la disminución de la calidad de vida de muchas familias ecuatorianas, si no se buscan herramientas 
para el fortalecimiento del tejido productivo y el fomento de la empleabilidad y el dinamismo productivo del país (Considerando del Decreto Ejecutivo 1074, 2020). Los sectores productivos y laborales más afectados han sido los del comercio al por mayor y al por menos (centros y locales comerciales) excepto los dedicados a la producción y expendio de alimentos y servicios de primera necesidad; el de la hotelería y turismo; los espectáculos públicos (entretenimientos y recreación); el inmobiliario; la industria manufacturera, entre otros.

Lo expresado se corrobora por el Observatorio de la Organización Internacional del Trabajo en la quinta edición referida al Covid-19 y el mundo del trabajo de 30 de junio 2020. Sostiene que el trabajo se ha visto muy afectado por la aplicación de medidas de confinamiento, en particular varias formas de cierre de lugares de trabajo, excepto los esenciales, siendo, en este sentido, la región las Américas la más afectada por restricciones a trabajadores y lugares de trabajo. Así, "la pandemia ha puesto de manifiesto amplias deficiencias y desigualdades en el mundo del trabajo, y las ha acentuado" (OIT, 2020), como en el caso de las mujeres, los jóvenes y los trabajadores del sector informal, lo que "pone de relieve tendencias inquietantes, susceptibles de exacerbar la disparidad y condicionar los modestos avances logrados en los últimos años en materia de igualdad de género en el mercado laboral" (OIT, 2020). Por lo que concluye que la pérdida de horas de trabajo en la primera mitad de 2020 ha sido mayor que la prevista, lo que pone de manifiesto el empeoramiento de la situación en las últimas semanas, en particular en los países en desarrollo" (OIT, 2020).

Por su parte, el Ministerio de Trabajo ha proporcionado las siguientes cifras respecto a las novedades referidas a las relaciones laborales, durante el estado de excepción. Así, se informa que a tres meses desde que se suspendió la jornada de trabajo presencial, 425.328 personas se encuentran laborando mediante la modalidad de teletrabajo (138.927 en el sector privado y 286.401 en el sector público). Que durante la pandemia se dio por terminado un total de 180.852 contratos ( 15.724 por despido intempestivo; otros 16.874 alegando fuerza mayor y 100.797 por acuerdo entre las partes; 47.457 terminaron por 
otras causales como desahucio, conclusión de obra, etc.). De lo dicho, se concluye que el $20 \%$ de las desvinculaciones se produjeron de modo imprevisto, orillando al desempleo a más de 30.000 personas en el país (Oficio Nro. MDT-MDT-2020-0335, citado en Decreto Ejecutivo 1074, 2020).

De esta manera las actividades repentinamente suspendidas, al menos las que no podían desarrollarse mediante la modalidad del teletrabajo, llevaron a empleadores, a pequeñas y medianas empresas a una situación económica extrema, en razón de que debían continuar pagando las remuneraciones y los demás beneficios de ley, así como los aportes al Instituto Ecuatoriano de Seguridad Social, pese a mantener las actividades paralizadas y consecuentemente no tener ingresos. Situación que en pocos meses se concretó en la terminación de las relaciones laborales, en algunos casos, motivada en el cierre y liquidación de algunas pequeñas y medianas empresas, como consecuencia de la imposibilidad de los trabajadores de cumplir con el objeto del contrato individual de trabajo y el descalabro económico de la parte empleadora.

\section{REGULACIONES DURANTE LA EMERGENCIA SANITARIA}

Frente al panorama devastador ya descrito, era necesario e imperativo arbitrar medidas para orientar el accionar en las actividades productivas y laborales en general a fin de garantizar, en primer lugar, la salud y seguridad de todas las personas trabajadoras y de la sociedad en general, $\mathrm{y}$, en segundo lugar, procurar el menor impacto posible en las relaciones laborales, y por ende en la economía de los hogares. Así, casi de forma inmediata, se activaron las propuestas de reformas en el sistema normativo. Pero, como es de suponer, lo primero fueron Acuerdos Ministeriales, que siempre son más ágiles en su expedición. En todo caso, fue una respuesta absolutamente reactiva frente a una crisis generalizada que obligaba una intervención estatal. Carlos Palomeque (2011) ya nos advertía, que la crisis económica ha acompañado siempre al Derecho del Trabajo como "un compañero de viaje histórico", puesto que "la lógica clásica del Derecho del Trabajo presupone verdaderamente la expansión o, al menos, la estabilidad del sistema económico" (p. 36). De allí que se entienda que 
las autoridades de trabajo no podían pasar por alto la crisis económica y sanitaria generalizada, como se evidencia de todas las regulaciones emergentes que se expidieron para hacerle frente, según veremos a continuación.

\subsection{Acuerdos ministeriales}

Fue el Ministerio del Trabajo, responsable de velar por los derechos de los trabajadores y empleadores, el que se pronunció expidiendo de manera emergente acuerdos ministeriales sobre medidas ya contempladas, por cierto, en el Código del Trabajo. Es decir, no se trató de nuevas regulaciones, sino de orientaciones para aplicar normativa ya existente. Concretamente nos referimos al teletrabajo emergente, así como, posteriormente, a las directrices para la aplicación de la modificación, reducción y suspensión de la jornada de trabajo, que pasaremos a revisar de inmediato.

\subsubsection{Teletrabajo emergente}

La modalidad del teletrabajo, entendida como una forma de prestación de servicios de carácter no presencial, haciendo uso de las tecnologías de la información y comunicación, ya se aplicaba tímidamente en el Ecuador. Se encontraba regulada por el AM MDT 190-2018. Decimos tímidamente, ya que hasta la crisis generada por la pandemia del Covid-19, eran muy pocos los empleadores que utilizaban esta modalidad, posiblemente por desconfianza, ya que existe, o al menos existía hasta antes de marzo de 2020, la idea en el imaginario colectivo, que si no existe una supervisión directa y permanente del trabajo no hay eficacia en los resultados ni un trabajo verdaderamente efectivo. Cabe citar al respecto el Art. 61 del Código de Trabajo (2005) que se refiere al cómputo del trabajo efectivo, entendido como aquel en que el trabajador se halla a disposición de sus superiores o del empleador, cumpliendo órdenes suyas. Esto, doctrinariamente se ha considerado, que no equivale a que el trabajador realice sin cesar una determina actividad, sino que incluye el tiempo en que aun no haciendo nada, se encuentra de todos modos a disposición del empleador; es decir, cumpliendo órdenes o dispuesto a cumplirlas. En todo caso, estimamos que no había la confianza y madurez necesaria como para permitir 
que las personas trabajadoras realicen sus actividades, lejos del "ojo" de la persona beneficiaria de los servicios o de sus supervisores.

En virtud del Decreto Ejecutivo 1074 (2020), ya referido, que ordenó la suspensión de las jornadas laborales presenciales, resultó obligado el acogerse, en la medida de lo posible, a esta modalidad del teletrabajo. Ante ello, el Ministerio del Trabajo expidió el 12-III-20 el AM MDT 0762020. Este Acuerdo reguló el teletrabajo de manera emergente en los términos que revisaremos enseguida. Lastimosamente, dada la emergencia, no fue posible verificar el cumplimiento previo de los requisitos para una adecuada implementación de esta modalidad, particularmente en cuanto a la provisión de los materiales de trabajo y verificación de las condiciones de salud y seguridad ocupacional. Otra gran desventaja, sin duda, ha sido que una gran cantidad de actividades no pueden realizarse a través de los medios telemáticos; o simplemente, no existían las condiciones mínimas, como es la necesaria conectividad y el desarrollo previo de competencias digitales. Sea como fuere, tanto trabajadores como empleadores debieron, pese a las resistencias, resignarse y aventurarse a incursionar en esta modalidad de trabajo, de manera, muchas veces, improvisada.

El Ministerio de Trabajo dejó claramente establecido que el acogerse o no a esta modalidad, era absolutamente potestativo del empleador. Esto en razón de la parte empleadora es la que tiene siempre la facultad de dirigir, organizar y supervisar el trabajo, y, por ende, es a la que le corresponde decidir, analizadas las circunstancias y tipo de actividad, si era o no procedente acogerse a esta modalidad laboral. En caso de hacerlo, debía simplemente informar al Ministerio sobre el inicio y el fin de la modalidad, con la indicación de los trabajadores que se sometían a la misma. Es importante destacar que, mediante este cambio emergente, solo se facultaba modificar el lugar del trabajo, no así el resto de las condiciones de trabajo. 
Esta modalidad, implementada como consecuencia del Covid-19, es decir, el teletrabajo "emergente" puede terminar, ya sea por acuerdo de las partes, en cualquier momento, o por la terminación de la declaratoria de emergencia sanitaria, que le dio origen. Más adelante revisaremos la reforma introducida, mediante la Ley de Apoyo Humanitario, por la que se incorpora al Código del Trabajo el teletrabajo como una modalidad más, ya no "emergente", en cuyo caso no procede, ha de entenderse, la terminación de la declaratoria de emergencia sanitaria como causal de finalización de dicha modalidad contractual.

\subsubsection{Modificación, reducción y suspensión de las jornadas de trabajo}

El Ministerio de Trabajo, días después, expidió el AM 077-2020-, de 15-III-20, solo para sector privado, para impartir directrices para la aplicación de la modificación, reducción y la suspensión de las jornadas de trabajo. La intención fue presentar a los empleadores las alternativas, ya previstas en el Código del Trabajo, que podían aplicar a fin de afrontar la emergencia provocada por el Covid-19. Estas medidas pueden subsistir hasta tanto acuerden las partes, de común acuerdo, ponerle término, o bien por la finalización de la declaratoria de emergencia.

Al respecto cabe recordar que existen axiomas jurídicos que podemos invocar para justificar ciertas normativas, disposiciones o acciones. Tal es el caso de los axiomas, por una parte, de que en Derecho "las cosas de deshacen como se hacen"; y por otra, aquel que nos dice que "quien puede lo más, puede lo menos". Es por ello que podríamos sostener que, si las partes estuvieron facultadas para acordar las condiciones de trabajo antes de iniciar la relación laboral, ellas mismas puedan modificarlas, en cualquier momento, siempre que en todo caso no implique regresión, ni renuncia de derechos. Los mismo podríamos decir respecto al segundo: si las partes pudieron acordar libremente las condiciones de la relación laboral (lo más), pueden modificar, de común acuerdo algunas de esa condición (lo menos). 
También en la doctrina se reconoce el ius variandi, entendido como la facultad que tiene el empleador de modificar la forma y modalidades del trabajo. Pero, para la validez de estas modificaciones es preciso la concurrencia de los siguientes requisitos que nos menciona Rosa Elena Bosio (2019), los mismos que están recogidos en la Ley del Contrato de Trabajo, y son: "la razonabilidad, la alteración no esencial del contrato y la ausencia de perjuicio material y moral para el trabajador" (p.169). Indudablemente que todos los requisitos mencionados pueden resultar subjetivos y muy cuestionables al momento de su aplicación. Bien se podría sostener que todas las medidas emergentes no han sido suficientemente razonables, permiten la alteración de manera esencial el contrato y perjudican material y moralmente al trabajador. No obstante, situaciones completamente excepcionales, ajenas a la voluntad de las partes, como son la declaratoria de la emergencia sanitaria y posterior declaratoria del estado de excepción y el consecuente toque de queda, como las que se viven a causa de la pandemia, no solo en el Ecuador sino a nivel mundial, legitiman en buena parte tales medidas, que son igualmente excepcionales y temporales.

Es por ello que la modificación de la jornada puede acordarse entre las partes vinculadas por un contrato de trabajo cuando se impone para continuar con el desarrollo de las actividades productivas y laborales. Es así como dentro de las directrices previstas en el AM-MDT 077-2020 (2020) se estableció que la jornada podía modificarse, incluyendo los días sábados y domingos, sin que por ello se considerara jornada extraordinaria, siempre que no excediera de las 40 horas semanales. Exigía además que se garantice el descanso de 2 días semanales, según la reforma introducida mediante AM-MDT 80-2020 (2020). También la modificación de la jornada puede darse al momento de aplicar la jornada de recuperación, prevista en el Art. 60 del Código del Trabajo, a la que nos referiremos más adelante, a propósito de la suspensión emergente de la jornada de trabajo. El AM-MDT 077-2020 (2020) simplemente ratifica lo ya establecido en el Código del Trabajo, al establecer que corresponde al empleador determinar la forma y el horario de trabajo, al retorno, respetando límites de jornada máxima. La novedad, en todo caso, está en la fijación de un límite semanal 
para la jornada de recuperación, situación que no contempla el Código de Trabajo, sino para la jornada suplementaria. Así, fija como máximo 12 semanales y 8 en sábados, según reforma introducida por el AM MDT 80-2020 (2020).

En cuanto a la reducción de la jornada laboral, cabe insistir en que esta igualmente ya se encuentra prevista en el Código del Trabajo (2005), como primer artículo enumerado a continuación del Art. 47 (es decir, como 47.1). La reducción excepcional de la jornada de trabajo es resultado de la reforma introducida por la Ley de Promoción del Trabajo Juvenil y regulación excepcional de la jornada de trabajo (RO-S 720, 28-III-2016, 2016, última modificación 1-IV-16). No obstante, dicha regulación limita esta reducción excepcional a seis meses, renovables por una sola vez. Además, exige que medie el previo acuerdo de las partes. Por su parte, el AM-MDT 077-2020 (2020) solo menciona la reducción de la jornada como una alternativa para mitigar la crisis, y en todo caso, para no llegar a la suspensión de las jornadas, y peor aún a la terminación de las relaciones laborales. Sin embargo, como veremos más adelante, la Ley de Apoyo Humanitario reguló de una manera diferente esta reducción de la jornada, con los efectos que comentaremos oportunamente.

Como tercera opción prevista en el AM-MDT 077-2020 (2020), se encuentra la suspensión de la jornada de trabajo, para cuando sea imposible, como medidas emergentes, el teletrabajo, la modificación, o la reducción de la jornada laboral. Dicha suspensión debía acogerse a lo previsto en el Art. 60 del Código del Trabajo, para lo cual el empleador determinará forma y horario (según se agregó de manera expresa el AM-MDT 0932020. 2020). Con todo, en el mismo Acuerdo se aclara que en caso de que el empleador decida acogerse a la suspensión de la jornada de trabajo, solo debía notificar al trabajador, sin que ello se considerara despido intempestivo. El referido artículo 60 se refiere al supuesto de que por causas ajenas a la voluntad de las partes se produjere una suspensión colectiva de las actividades, en cuyo caso el empleador tiene derecho a recuperar las horas no trabajadas, una vez que se retorne a los lugares de trabajo. (CT, 2005) Para ello podrá fijar nuevos horarios de trabajo, extendiendo las jornadas hasta por tres horas diarias, hasta 
completar el tiempo perdido y la remuneración pagada. Esto en razón de que durante la suspensión persiste la obligación del empleador de pagar la remuneración a los trabajadores que se ven impedidos de prestar sus servicios de manera regular.

\subsubsection{Calendarización para pagos}

El principal derecho de las personas trabajadoras es percibir, como contraprestación del servicio prestado, las remuneraciones y demás beneficios económicos de manera íntegra y oportuna. Consecuentemente, el pago de las obligaciones pecuniarias es la principal obligación de la parte empleadora. Tal es así, que la disminución, la falta de pago o el retraso en el cumplimiento de dicha obligación, constituye una causa para que los trabajadores soliciten el visto bueno. En caso de que, por este motivo, y mediando la respectiva resolución del Inspector del Trabajo, se diera por terminadas las relaciones laborales, el empleador deberá las correspondientes indemnizaciones por tratarse de un despido indirecto. Otra consecuencia de la inobservancia de los principios mencionados es la condena al empleador al pago del triple del monto adeudado, si para el cobro de las remuneraciones impagas, el trabajador tuviera que acudir a la vía judicial; o tratándose del fondo de reserva, la condena al pago del recargo del 50\%; a más de multas que obviamente por tales motivos pudieran imponer las autoridades del trabajo, multas que oscilan desde los tres hasta los veinte salarios básicos.

Pese a todo lo dicho, resulta indudable que la crisis provocada por el Covid-19, puso a los empleadores en situaciones extremadamente difíciles y excepcionales, impidiéndoles cumplir con sus obligaciones laborales. De allí que para evitar ya sea la terminación de los contratos de trabajo, y peor aún, el cierre o liquidación de las empresas, el AM MDT 80-2020, de 28 de marzo de 2020, permitió que las partes de una relación laboral acordaran un calendario para honrar las obligaciones pendientes de pago. Esta calendarización, sin duda, contradice los principios de oportunidad y oportunidad. No obstante, estimamos que, dadas las circunstancias absolutamente excepcionales, y siempre que se garantizara a los trabajadores un ingreso mínimo, digno y suficiente, según sus ingresos 
acostumbrados, podía darse paso a esta alternativa que dio el Ministerio de Trabajo de acordar un calendario de pagos, hasta tanto se regularizara medianamente la situación producto de la emergencia.

\subsubsection{Regulación emergente de vacaciones}

Otro de los derechos reconocidos a los trabajadores sujetos al Código del Trabajo (2005) es el de disfrutar de un período de 15 días de vacaciones por cada año de servicios. Este derecho se incrementa en un día por cada año que excede a los 5 primeros laborados en favor del mismo empleador, hasta un máximo de 15 días adicionales. Este derecho, como todos los demás de los trabajadores, es irrenunciable. Este período de vacaciones, de no pactarse en el contrato, o de no mediar acuerdo de las partes, es facultad del empleador el fijarlo, siempre que lo comunique el trabajador con al menos 3 meses de anticipación.

Lo dicho son las reglas generales, de conformidad a las normas vigentes previstas en el Código del Trabajo. Sin embargo, frente a la emergencia sanitaria, y dada las dificultades económicas de los empleadores, sumado al hecho de que algunas actividades, tales como las referidas a espectáculos públicos, cines, discotecas, restaurantes, entre otras, estaban expresamente prohibidas, el AM-MDT 80-2020 (2020) prescribió la posibilidad realizar una planificación emergente de las vacaciones. Dicha planificación era facultativa de la parte empleadora, y no requería observar la anticipación legal. Es decir, se podía simplemente notificar al trabajador que debía acogerse al goce de sus vacaciones acumuladas, prácticamente, de manera inmediata. Y, en caso de que mediara acuerdo de las partes, se podían otorgar, inclusive, vacaciones anticipadas. Con esta medida, no muy comprendida ni aceptada, se pretendió, al menos de manera emergente, atenuar el impacto de la suspensión de las actividades. Muchos no veían la diferencia con la suspensión de la jornada ya que tanto esta como las vacaciones implican que el empleador debe remunerar al trabajador por el tiempo no laborado. En el primer caso, condicionado a que recupere las horas no trabajadas. En el 
segundo, sin recuperación, pero en cambio resultaba un alivio por cuanto las vacaciones acumuladas requieren contar con la debida provisión presupuestaria.

\subsection{Ley de Apoyo Humanitario}

Frente a los efectos negativos en la economía mundial producidos por la pandemia del Coronavirus, era necesario dar un "alivio a la ciudadanía, al sector productivo y a la economía popular y solidaria para hacer frente a la actual situación económica y sanitaria del país" (Ley de Apoyo Humanitario para combatir la crisis sanitaria derivada del Covid-19, 2020). De conformidad a su Art. 1 de la Ley Humanitaria (2020), tiene por objeto establecer medidas de apoyo humanitario, necesarias para enfrentar las consecuencias derivadas de la crisis sanitaria ocasionada por el COVID-19, a través de medidas tendientes a mitigar sus efectos adversos dentro del territorio ecuatoriano; que permitan fomentar la reactivación económica y productiva del Ecuador, con especial énfasis en el ser humano, la contención y reactivación de las economías familiares, empresariales, la popular y solidaria, y en el mantenimiento de las condiciones de empleo. Así, el Capítulo III está dedicado a las medidas para apoyar la sostenibilidad del empleo. En él se regulan: los acuerdos de preservación de fuentes de trabajo (Arts. 16-18); el contrato especial emergente (Art. 19); la reducción emergente de la jornada de trabajo (Art.20); el goce de las vacaciones (Art. 21); temas que analizaremos de inmediato. El mencionado Capítulo se refiere además a: las prestaciones del seguro de desempleo (Art. 22-23); la priorización de contratación a trabajadores, profesionales, bienes y servicios de origen local (Art. 24); y, la estabilidad de trabajadores de la salud (Art.25), los mismos que no analizaremos en esta oportunidad por cuanto escapan del ámbito delimitado en la primera parte de este artículo.

\subsubsection{Acuerdos para la preservación del empleo}

Con la finalidad de preservar las fuentes de trabajo y garantizar estabilidad a los trabajadores, se podrán modificar, previo acuerdo de las partes, las condiciones económicas del contrato de trabajo (Ley de Apoyo Humanitario para combatir la crisis sanitaria derivada del Covid-19, 2020, arts. 16-18). Tales 
acuerdos pueden ser a propuesta de cualquiera de las partes. Serán bilaterales y directos, es decir entre el empleador en cuestión y sus trabajadores, individualmente considerados. No obstante, si las nuevas condiciones económicas fueran resueltas por la mayoría de los trabajadores, quienes no estuvieren de acuerdo, de todos modos, quedarán obligados a acatarlas.

Los acuerdos a los que se arriben bajo ninguna circunstancia podrán afectar el salario básico o los salarios sectoriales determinados para jornada completa o su proporcionalidad en caso de jornadas reducidas. Esto debe entenderse, que no se admitirá, a pretexto de la emergencia sanitaria, o la necesidad de preservar la fuente de trabajo, que se acuerden salarios por debajo de los mínimos establecidos por el Ministerio de Trabajo. Sin embargo, puede resultar por efecto del acuerdo que a una persona trabajadora que recibía el salario mínimo, se le reduzca la jornada y consecuentemente su ínfima remuneración también, de manera proporcional. Ante ello cabe cuestionarse si resulta legítimo que se reduzcan los mínimos, considerados tales para la supervivencia, o si acaso se debió establecer una tabla para que las reducciones afecten a quienes más ganan y no a quienes tienen los niveles más bajo de ingresos.

En razón de que resulta casi impensable que los trabajadores acepten acordar con sus empleadores la reducción de sus ingresos, se ha previsto una norma que establece que, si fuere imprescindible para subsistencia de la empresa tales acuerdos y no se los lograre, entonces se deberá dar paso al proceso de liquidación. Es decir, es una clara forma de presionar a los trabajadores a aceptar esta violación a sus derechos con el único propósito de salvaguardar la fuente de empleo. Es de esperar, que los acuerdos sean de los más mesurados, razonables, de corta duración, y que se den cuando se justifique que verdaderamente es la única medida para evitar la liquidación de la empresa o negocio.

Para que procedan tales acuerdos el empleador debe presentar los estados financieros de forma clara y completa que justifiquen la necesidad de suscribirlos. En todo caso, deberá entenderse que estos acuerdos pueden pactarse 
mientras dure la emergencia y por tan solo el tiempo fijado por las partes; más aún estimamos que faltó delimitar, al igual que si se lo hizo para el caso de la reducción de la jornada y el contrato especial emergente, a un año con la posibilidad de una sola renovación por un período igual. En todo caso, una vez suscritos tales acuerdos, estos tendrán preferencia sobre cualquier otro acuerdo o contrato. Ello sin perjuicio de que puedan ser impugnados por terceros en caso de fraude. Y, si el juez presume la existencia de un delito vinculado a la celebración del acuerdo, lo dará a conocer a la Fiscalía General del Estado para las investigaciones y acciones correspondientes. Así también, el uso doloso de recursos de la empresa en favor de sus accionistas o administradores, durante la vigencia del acuerdo, será considerado causal de quiebra fraudulenta y dará lugar a la anulación del acuerdo y a la sanción establecida por el Código Orgánico Integral Penal. Finalmente, para evitar que los empleadores se beneficien maliciosamente de los acuerdos (reduciendo los ingresos de sus trabajadores y elevando consecuentemente sus ganancias, o al menos reduciendo sus costos), se establece la prohibición de distribuir dividendos correspondientes a los ejercicios en que los acuerdos para la preservación de los empleos estén vigentes, ni reducir capital social durante tiempo de vigencia de los mismos. En todo caso, corresponderá al Ministerio del Trabajo supervisar el cumplimiento de los referidos acuerdos. Y, de producirse el despido del trabajador al que se aplica el acuerdo, dentro del primer año de vigencia de la Ley de Apoyo Humanitario, las indemnizaciones correspondientes se calcularán con la última remuneración percibida por el trabajador antes del acuerdo, de conformidad al Art. 95 del Código del Trabajo, es decir, considerando todo lo que el trabajador reciba en dinero, servicios o especies, inclusive lo que percibiere por trabajos suplementarios o extraordinarios, a destajo, comisiones, participación en beneficios o cualquier otra retribución que tuviera el carácter de normal en la industria o servicio. 


\subsubsection{Contrato especial emergente}

La figura del contrato especial emergente fue introducida por la Ley de Apoyo Humanitario para contribuir a la sostenibilidad del empleo. Se considera tal al contrato individual de trabajo por tiempo definido que se celebra para la sostenibilidad de la producción y fuentes de ingresos en situaciones emergentes o para nuevas inversiones o líneas de negocio, productos o servicios, ampliaciones o extensiones del negocio, modificación del giro del negocio, incremento en la oferta de bienes y servicios por parte de personas naturales o jurídicas, nuevas o existentes o en el caso de necesidades de mayor demanda de producción o servicios en las actividades del empleador. Es decir que se trata de un contrato emergente por tiempo definido de un año renovable por una sola vez.

En este contrato especial es posible pactar una jornada laboral completa o parcial, repartida hasta en seis días semanales. Consecuentemente, la remuneración y demás beneficios legales deberán ser pagados de manera proporcional, de acuerdo con la jornada pactada. En caso de terminación antes del plazo o de manera unilateral por cualquier de las partes, da derecho a la bonificación por desahucio y demás beneficios de ley calculados de conformidad al Código del Trabajo. Superado el plazo, de continuar, se convierte en indefinido. El Ministerio de Trabajo, mediante AM 132-2020, de 15 de julio expidió las directrices para el registro de las modalidades y acuerdos laborales establecidos en la Ley de Apoyo Humanitario. Se aclara, mediante la Disposición Transitoria Tercera que los acuerdos suscritos con anterioridad, de conformidad con las directrices del mismo Ministerio, tendrán plena vigencia por el tiempo que se lo hubiere pactado y se podrán renovar por el mismo plazo por una sola ocasión adicional. Con todo, se advierte que en cualquier momento se podrán suscribir nuevos acuerdos según la Ley de Apoyo Humanitario.

Por lo tanto, las novedades que trae este contrato, a más de retornar a la figura del contrato a plazo, ya derogado desde el 20-IV-15, es que la jornada parcial permanente tiene un mínimo 20 horas y que el máximo de 40 horas semanales puede ser repartido en máximo seis días semanales. Además, 
que el descanso semanal será al menos de veinticuatro horas consecutivas. Tampoco habría lugar a las indemnizaciones por terminación del contrato antes del plazo, sino solo a la liquidación correspondiente y a la bonificación del 25\% por cada año de servicios. Ha de entenderse que, superado el plazo máximo de duración de este contrato, es decir, de dos años, y se diere por terminado, entonces sí habrá lugar a las indemnizaciones que correspondan al contrato indefinido que es al momento la modalidad típica de los contratos de trabajo. Dicha indemnización sería equivalente a tres meses de remuneración, si el contrato no supera los tres años de duración, o bien, a un mes por cada año de servicios a partir del cuarto año (considerando la fracción del año como año completo). Toda indemnización, en todo caso, se calculará en función del Art. 95 primer inciso del Código del Trabajo (2005), como ya ha quedado descrito anteriormente

\subsubsection{Reducción emergente de la jornada de trabajo}

Ya habíamos señalado que en el Código del Trabajo se incorporó, en el Art. 47.1, la regulación excepcional de la jornada de trabajo. No obstante, resulta imperioso advertir que la Ley de Apoyo Humanitario no deroga dicho artículo, ni la referida figura. Es decir que al momento coexiste la reducción excepcional de la jornada de trabajo, con la reducción emergente de la jornada de trabajo. La primera, insistimos, regulada por el Código del Trabajo, que permite la reducción de la jornada hasta en un $25 \%$, con la consecuente reducción de la remuneración. Para que proceda se requiere contar con la autorización del Inspector del Trabajo, el acuerdo de las partes, y la justificación de las circunstancias que obligan tal medida. En todo caso, dicha reducción solo puede ser hasta por seis meses renovable por tan solo una vez.

En cambio, por medio de la Ley de Apoyo Humanitario (2020), se introdujo mediante el Art. 20 la figura de la reducción emergente de la jornada de trabajo. Se entiende esta nueva figura como la rebaja de las horas de trabajo, en este caso hasta un $50 \%$, con la consecuente reducción, igualmente proporcional de la remuneración (aunque se prohíbe que se reduzca más del $45 \%$, es decir la remuneración no podrá ser inferior al 55\%). 
Igualmente, los aportes al Instituto Ecuatoriano de Seguridad Social se realizarán en función de la jornada reducida. En este sentido mejoró la disposición del Código del Trabajo que establece que, pese a la reducción de la remuneración, como consecuencia de la reducción de la jornada, los aportes al Instituto Ecuatoriano de Seguridad Social que le corresponde al empleador deben realizarse sobre ocho horas de trabajo. Permite, además, separándose del límite fijado en el Código del Trabajo, que esta medida sea hasta por un año renovable por uno más.

Ha de entenderse que por ser emergente solo aplica como medida para salvaguarda las fuentes de empleo, amenazada por la emergencia sanitaria provocada por el Covid-19. Es decir, es una facultad del empleador en situaciones de caso fortuito o fuerza mayor, debidamente justificado. De producirse esta reducción emergente de la jornada de trabajo es obligación del empleador notificar al Ministerio de Trabajo respecto al período de reducción y nómina de trabajadores afectados. Además, para evitar que se burlen mayormente los derechos de los trabajadores, en caso de despido los cálculos de las indemnizaciones se los realizará en función de la última remuneración percibida por el trabajador anterior a la reducción de la jornada, en la forma ya señalada anteriormente, según el Art. 95 del Código del Trabajo.

Por último, se establecen igualmente algunas prohibiciones adicionales a los empleadores. Mientras dure la implementación de la reducción de la jornada, los empleadores no podrán reducir capital social de la empresa ni repartir dividendos obtenidos en los ejercicios en que esta jornada esté vigente. Los dividendos serán reinvertidos en la empresa, mediante el correspondiente aumento de capital.

Mediante Acuerdo 133, de 15 de julio de 2020, el Ministerio de Trabajo (2020) expidió las directrices para la aplicación de la reducción emergente de la jornada de trabajo establecida en la Ley de Apoyo Humanitario. Este Acuerdo no hace sino ratificar absolutamente el Art. 20 de la Ley en referencia. Las únicas diferencias son las innecesarias menciones, por una parte, de lo que habrá de entenderse por 
caso fortuito o fuerza mayor. Ejemplifica indicando que se trata de aquellos casos en donde existan imprevistos imposibles de prever o generen imposibilidad de realizar el trabajo con normalidad y en consecuencia se deba reducir la jornada laboral ordinaria. Y, por otra, sobre la proporcionalidad para el cálculo de los beneficios económicos, que se los pagará en función de la jornada reducida. Decimos que se trata de menciones innecesarias puesto que ya la propia Ley de Apoyo Humanitario (2020), a través de la Disposición interpretativa indicó cómo deberá entenderse el numeral 6 del Art. 169, y la aplicación del principio de proporcionalidad para el caso de las remuneraciones, y demás beneficios de ley, está prevista en el Art. 82 del Código del Trabajo. Lo importante, en todo caso, es que este Acuerdo es coherente con lo que ya habíamos anotado, en el sentido de que la reducción de la jornada prevista en el Art. 20 de la Ley de Apoyo Humanitario, es diferente, e independiente, de la prevista en el Código del Trabajo en el Art. 47.1. Así, el Art. 8 del Acuerdo en cuestión, establece que no se podrá aplicar la disposición del Art. 20 a los trabajadores que ya se encuentren con una jornada reducida de conformidad al Código del Trabajo, mientras esta esté vigente.

\subsubsection{Goce de vacaciones}

Siguiendo con el análisis de las medidas previstas en la Ley de Apoyo Humanitario para salvaguardar las fuentes de empleo, tenemos la regulación respecto al goce de las vacaciones a las que tienen derecho los trabajadores. Así, en su Art. 21 establece que unilateralmente el empleador podrá, durante dos años a partir de la vigencia de la referida Ley, notificar al trabajador con el cronograma de vacaciones; o, establecer la correspondiente compensación económica de días de inasistencia como vacaciones devengadas. El AMMDT 80-2020 (2020) ya revisado, también se refirió al goce de las vacaciones y le confirió al empleador la facultad de decidir unilateralmente sobre el período de las vacaciones y de notificarlas sin el tiempo requerido por el Código del Trabajo. No obstante, la Ley de Apoyo Humanitario (2020) adicionalmente fija el tiempo máximo por el cual aplica esta medida. En todo caso, estimamos que una vez más, se afectan los derechos de los trabajadores, privándoles de la facultad de 
acordar con su empleador el período de sus vacaciones o de acumularlas, puesto que se le faculta al empleador para que decida unilateralmente, no solo el cronograma de vacaciones sino también la imputación, con cargo a vacaciones, de los días de inasistencia del trabajador. Pese a ello, podemos sostener que tal afectación es mínima, comparada con otras que se han dado con motivo de la emergencia sanitaria. Decimos que es mínima por cuanto si el cronograma de vacaciones fuera notificado con por lo menos tres meses de anticipación, sería lo que ya constaba en el Código del Trabajo. Así mismo, el imputar las inasistencias a vacaciones, tampoco resulta muy gravoso, dado que de esta manera no se le descontaría al trabajador el día de inasistencia, sino por el contrario, se le remuneraría como vacación; aunque ello implique que se le reducen los días ininterrumpidos de goce de vacaciones a los que tiene derecho.

\subsubsection{Reformas al Código del Trabajo}

\subsubsection{Teletrabajo}

Ya habíamos comentado que la modalidad del teletrabajo estaba regulada por el AM-MDT 190-2018 y posteriormente, durante la emergencia, se lo reguló como teletrabajo emergente, mediante el AM MDT 076-2020. Ahora, como consecuencia de la Primera Disposición Reformatoria de la Ley de Apoyo Humanitario se incorporó esta figura al Código del Trabajo, a continuación del Art. 16. Es decir, luego de los contratos por obra cierta, por tarea y a destajo, por lo que se le está considerando como un tipo de contrato según la forma de ejecución.

Se define al teletrabajo como una forma de organización laboral, que consiste en el desempeño de actividades remuneradas o prestación de servicios utilizando como soporte las tecnologías de la información y la comunicación para el contacto entre el trabajador y la empresa, sin requerir la presencia física del trabajador en un sitio específico de trabajo. En esta modalidad el empleador ejercerá labores de control y dirección de forma remota y el trabajador reportará de la misma manera (Ley de Apoyo Humanitario, 2020, Primera Disposición Reformatoria). Puede revestir una de las siguientes formas: 
autónomo: cuando se utiliza el propio domicilio o un lugar escogido para desarrollar la actividad profesional, puede ser una pequeña oficina, un local comercial. En este tipo se encuentra la persona que trabaja siempre fuera de la empresa y sólo acude a la oficina en algunas ocasiones. Móvil: cuando el teletrabajador no tiene un lugar de trabajo establecido y cuyas herramientas primordiales para desarrollar sus actividades profesionales son las tecnologías de la información y la comunicación, en dispositivos móviles. Parcial: cuando el teletrabajador labora dos o tres días a la semana en su casa y el resto del tiempo lo hacen en una oficina. Ocasional: cuando el teletrabajador realiza sus actividades en ocasiones o circunstancias convenidas.

Se podrá pactar la modalidad del teletrabajo al inició de la relación laboral o con posterioridad. En todo caso, el empleador tiene la obligación de informar a la autoridad del trabajo de la vinculación de teletrabajadores.

Con ocasión de la regulación del teletrabajo, se introduce de manera adicional un nuevo derecho que ha surgido en los últimos años, como consecuencia de los avances de las tecnologías de la información y de la comunicación. Nos referimos al derecho a la desconexión, entendido como el tiempo durante el cual el teletrabajador no está obligado a responder comunicaciones, órdenes u otros requerimientos del empleador. Al respecto Pérez (2019) nos dice que:

La revolución digital y los procesos de automatización y globalización han dado lugar a un fenómeno de conectividad permanente que está afectando a todos los ámbitos de la actividad humana, incluido el laboral donde las fronteras entre tiempo de trabajo y descanso se están diluyendo cada vez más.

Este incremento de la comunicación continua,impulsada por el empleo de dispositivos tecnológicos como herramientas para el desarrollo de la prestación de servicios laborales, propicia que muchos trabajadores se encuentren permanentemente "enganchados" al trabajo, lo que ha dado paso a lo que denomina "hiperconexión", lo que exige una pronta y adecuada regulación (Pérez, 2019). Consecuentemente, el derecho a la desconexión 
supone que el trabajador podrá literalmente "desconectarse" de su trabajo, al menos por un período de 12 horas diarias, así como durante los fines de semana, feriados, permisos o vacaciones. Es decir, que a pretexto de los medios de interconexión con los que se dispone actualmente, y que son herramientas propiamente de trabajo, no se puede afectar el derecho todo trabajador al ocio y al descanso, y a destinar su tiempo libre a las actividades a quien bien tuviere, ya sea académicas, profesionales, familiares, deportivas, espirituales, etc. Descanso que debe ser reparador para garantizar la salud además de la adecuada motivación para el trabajo, razón por la cual de ello no solo se beneficia el trabajador sino el empleador por cuanto va a redundar en una más efectiva productividad.

\subsubsection{Calificación del Covid-19 como enfermedad profesional}

El Derecho del Trabajo y el de la Seguridad Social protegen a la persona trabajadora por riesgos del trabajo desde el primer día de sus labores. Se entiende riesgos laborales a los accidentes de trabajo y a las enfermedades profesionales. Ambos son eventualidades a las que están sujetos los trabajadores con motivo o con ocasión de trabajo que realizan. En caso de producirse tales eventualidades, los trabajadores tienen derecho a recibir por parte del Instituto Ecuatoriano de Seguridad Social la asistencia médica, quirúrgica, farmacéutica, a los servicios de prótesis y ortopedia y a subsidios en dinero, es decir a recibir pensiones por incapacidad parcial o total, según corresponda. En caso de no estar afiliado es el empleador quien debe cubrir todos los gastos. Si se produjera la muerte de la persona trabajadora como consecuencia de la enfermedad profesional, sus deudos tendrán derecho a percibir una indemnización equivalente al sueldo o salario de cuatro años.

A propósito de los numerosos contagios por parte de trabajadores en general, y de personal del área de la salud en particular, se dio paso a la discusión y análisis de si acaso debía o no considerarse en todos los casos al Covid-19 como una enfermedad profesional. Inicialmente, fue el Ministerio del Trabajo el que se pronunció mediante Resolución 022 de 28 de 
abril de 2020. Sin embargo, de manera inmediata se rectificó dicha resolución, y así, al día siguiente, se expidió la Resolución 022 por medio de la cual se estableció que: la enfermedad del coronavirus (Covid-19) no constituye un accidente de trabajo ni una enfermedad profesional, a excepción de aquellos casos en los que se pudiera establecer, de forma científica y por métodos adecuados a las condiciones y a las prácticas nacionales, un vínculo directo entre la exposición a agentes biológicos que resulte de las actividades laborales realizadas por el trabajador. Posteriormente, se aprovechó la expedición de la Ley de Apoyo Humanitario para incorporar una reforma adicional al Código del Trabajo. Así, por medio de la Disposición Reformatoria Segunda de la mencionada Ley, se añade al final del artículo 363 del Código del Trabajo, que se refiere a la clasificación de las enfermedades profesionales, un nuevo numeral referido a la categoría: de síndromes respiratorios agudos causados por virus, para el caso de médicos, enfermeras, mozos de anfiteatro, de los departamentos de higiene y salubridad, sean del Estado, o de cualquier otra entidad de derecho público, o de derecho privado con finalidad social o pública, o particulares. (Ley de Apoyo Humanitario, 2020) Con ello se ha delimitado claramente que solo los profesionales y personal del área de la salud que fueren contagiados, con motivo o con ocasión del ejercicio de sus funciones y realización de sus actividades laborales, se puede considerar enfermedad profesional.

\subsubsection{Disposición interpretativa sobre el caso fortuito o fuerza mayor}

La causal de caso fortuito y fuerza mayor fue reiteradamente invocada por decenas de empleadores para poner fin a las relaciones laborales durante el estado de excepción por calamidad pública declarado en el Ecuador en el mes de marzo de 2020. La decisión la justificaban en la afectación sufrida por la emergencia sanitaria provocada por el Covid-19, y los consecuentes impactos en el ámbito laboral, especialmente con la suspensión de la jornada de trabajo, que obligaba en todo caso a seguir pagando las remuneraciones y aportes al Instituto Ecuatoriano de Seguridad Social, pese a la paralización absoluta de las actividades. Sin duda que muchos 
casos resultaban evidentes y justificados, pero se dieron muchos despidos masivos que obligó a delimitar, vía una disposición interpretativa, el alcance de la causal 6 del Art. 169. El Art. 169 se refiere a las causas por las cuales puede un contrato individual de trabajo terminar. El numeral 6 del referido artículo dice: por caso fortuito o fuerza que imposibiliten el trabajo, como incendio, terremoto, tempestad, explosión, plagas del campo, guerra $\mathrm{y}$, en general, cualquier otro acontecimiento extraordinario que los contratantes no pudieron prever o que previsto, no lo pudieron evitar.

Por medio de la disposición interpretativa, al referido numeral 6 del artículo 169 del Código del Trabajo (2005), se le dio el siguiente sentido:

En estos casos, la imposibilidad de realizar el trabajo por caso fortuito o fuerza mayor estará ligada al cese total y definitivo de la actividad económica del empleador, sea persona natural o jurídica. Esto quiere decir, que habrá imposibilidad cuando el trabajo no se pueda llevar a cabo tanto por los medios físicos habituales como por medios alternativos que permitan su ejecución, ni aún por medios telemáticos.

Somos del parecer, no obstante, que esta ley interpretativa no resulta suficiente. Sostenemos que no es suficiente, primero por lo tardía, y segundo, porque no considera otras opciones intermedias, como podría ser la posibilidad de dar paso a una suspensión total. Por suspensión total nos referimos a la paralización de las actividades laborales sin pago de remuneración, al igual que lo que sucede en el caso de un paro. Es decir, dicha paralización podría ser por el tiempo, previamente acordado entre las partes, siempre que no supere un año (al igual que el resto de situaciones reguladas en el Código del Trabajo como suspensiones del contrato individual de trabajo). Con ello se hubieran evitado muchas liquidaciones de empresas y negocios, que se han visto asfixiados económicamente al tener que seguir pagando todas sus obligaciones, pese a encontrarse paraliza la actividad productiva o laboral. 
En todo caso, cuando un juez determine que el empleador invocó de manera injustificada la causal de fuerza mayor o caso fortuito para terminar una relación laboral, se aplicará la indemnización por despido intempestivo prevista en el artículo 188 del Código del Trabajo (2005) multiplicada por uno punto cinco (1.5), (2do inc. Art. 17); es decir, si el trabajador hubiere laborado hasta tres años, percibirá por concepto de indemnización un monto equivalente a tres meses de remuneración; si hubiere trabajado más de tres años percibirá una remuneración por cada año de servicios. Consideramos acertado el condenar al empleador inescrupuloso con un recargo del $50 \%$ del monto de las indemnizaciones cuando alegando injustificadamente la causal 6 del Art.169 pone fin a la relación laboral. No es de ninguna manera admisible que se valgan de la emergencia sanitaria y la crisis económica provocada por el Covid-19, para reducir personal o despedir a trabajadores que tenían muchos años de servicios, con el claro propósito de evitar indemnizaciones, y aun, eventuales jubilaciones a cargo del empleador. El juez, como única autoridad competente, será el responsable de calificar si efectivamente la causa de la liquidación del negocio, y por ende de la terminación de las relaciones laborales, se debieron a la situación derivada del Covid-19.

\section{INOBSERVANCIA DE PRINCIPIOS DEL DERECHO DEL TRABAJO}

Hemos comentado las regulaciones, muchas veces erráticas, que por motivo de la crisis sanitaria fueron necesarias. Hemos también anticipado que muchas de ellas han sido claramente violatorias principios laborales; concretamente, a los principios de intangibilidad, no regresividad, progresividad, irrenunciabilidad; además de los de oportunidad e integridad referidos a los derechos económicos de los trabajadores. Fundamentemos nuestra aseveración, pasando breve revista a cómo cada uno de los referidos principios han sido flagrantemente inobservados de manera sistemática.

El principio de intangibilidad implica que los derechos de la persona trabajadora no pueden ser desconocidos ni desmejorados por ninguna ley, convenio o contrato colectivo. 
Es decir que los derechos laborales ya reconocidos y adquiridos por los trabajadores no pueden ni deben tocarse, razón por la cual sirven como un mínimo a partir de los cuales las normas posteriores deberán mejorar las condiciones, pero nunca desmejorarlas. Al decir de Andrés Páez (2019), se entiende por intangibilidad de los derechos laborales "que los mismos no pueden ser conculcados, cuando de forma definitiva e irrevocable se han incorporado al patrimonio del trabajador $y$, una vez conformado el derecho subjetivo, este no puede ser modificado por nuevas legislaciones o por cualquier cambio posterior" (p. 60). No obstante, la reducción emergente de la jornada de trabajo, prácticamente igual a la que consta en el Art. 47.1 del CT -reducción excepcional de la jornada de trabajo-, con la consecuente reducción de la remuneración y demás beneficios económicos, es claramente contraria a este principio.

Los principios de progresividad y no regresividad implican, según Corsini:

La garantía de la conquista de un estadio [...] asegurando un mínimo de dignidad existencial a los trabajadores, es decir, funciona como una válvula del sistema, que no permite que se pueda retroceder en los niveles de conquista protectorias logrados, e impide el retroceso a condiciones propias de períodos históricos que registran un mayor grado de desposesión. (citado por Páez, 2019, p. 61)

Por ello el mismo Páez (2019) concluye que "la noción de progresividad impide que se retroceda sobre lo que, hasta el momento se ha alcanzado, en materia de derechos laborales, entendiendo por retroceso un empeoramiento de los mismos" (p. 62). Aunque resulte obvio que en épocas de crisis económicas no se puede pretender incrementos o mejoras de los beneficios económicos, es precisamente en esas mismas épocas en las que se debe garantizar estabilidad laboral y económica a las personas que viven de sus ingresos fruto de un contrato de trabajo. En épocas de crisis, los trabajadores, ya parte débil de una relación laboral, se encuentran en situaciones de mayor vulnerabilidad a quienes es necesario garantizarles protección y el no desmedro de sus derechos. No obstante, se 
han inobservado principios fundamentales del Derecho del Trabajo que han mermado la protección a la parte trabajadora. Se ha dado una regresión de derechos el permitir por ejemplo que el empleador, unilateralmente, resuelva la disminución de la jornada laboral, el cronograma de vacaciones, la contratación a plazo fijo a través de la figura del contrato especial emergente (aunque en este caso ha de entenderse que aplica solo para los nuevos contratos). Todo ello, por supuesto, sin desconocer que es necesario asegurar las fuentes de empleo, y por ende flexibilizar las regulaciones a fin de que los empleadores, si acaso no aumenten, al menos mantengan los puestos de trabajo, en lugar de optar por el cierre o la liquidación de la empresa o negocio. La contracción de la economía ha impactado fuertemente a todos los sectores, pero, como siempre, los más afectados son quienes menos tienen y aquellos que dependen absolutamente de sus remuneraciones.

El principio de la irrenunciabilidad, impide que los trabajadores renuncien a sus derechos, en virtud de que se trata de la parte débil de una relación laboral, y por ende muy vulnerable frente a eventuales presiones o abusos por parte de los empleadores. Rosa Elena Bosio (2019) sostiene que cuando un trabajador renuncia a sus derechos es por "falta de capacidad de negociación o ignorancia, conminado por la desigualdad jurídica-económica existente con el empleador, con el objeto de preservar su fuente laboral", por lo que la renuncia en tales casos carecería de validez" (p. 25). Por ello, los acuerdos para la preservación de las fuentes de trabajo previsto en la Ley de Apoyo Humanitario, en los casos que se los haya suscrito, claramente adolecen de un vicio del consentimiento que es la fuerza. No cabe la menor duda de que los trabajadores se han visto constreñidos a aceptar condiciones en franca desmejora de las condiciones económicas, con el único afán de mantener un ingreso, aunque escuálido. Los trabajadores se han visto forzados a renunciar, expresa o implícitamente a varios de sus derechos y resignarse para conservar su puesto de trabajo. Nos referimos a los derechos a mantener su remuneración íntegra e inalterable; a mantener su jornada de trabajo completa (ya sea de ocho horas diarias o la parcial que hubieren acordado al inicio de la relación laboral), aun cuando de todos modos 
implique seguir trabajando tanto a más que antes; a su derecho a la intimidad familiar y el derecho al ocio, cuando se ha visto obligado a trabajar desde su casa, aún sin contar con los equipos ni medios necesarios, a toda hora, inclusive los días de descanso.

Los principios de integridad y oportunidad se refieren a la necesidad de que la remuneración llegue a cada trabajador de manera completa y a tiempo, es decir, conforme al monto y tiempo de pago acordados y conforme a la ley. Por lo tanto, el monto no puede ser de ninguna manera inferior al básico y los plazos para los pagos no pueden ser mayor a una semana o de un mes, según se refiera a salario o sueldo, respectivamente. Sin embargo, reiteramos, el permitir, por una parte, la calendarización de pagos de las remuneraciones y por otra la reducción de la remuneración, se han inobservado flagrantemente tales principios, aun cuando tales medidas pudieron estar justificadas en una situación absolutamente excepcional, y con el propósito de evitar un mal mayor, como sería la terminación de las relaciones laborales, o incluso la liquidación de los negocios.

Al respecto hacemos eco de las declaraciones del Observatorio de la Organización Internacional del Trabajo en su quinta edición dedicada al Covid-19 y el mundo de trabajo, de 30 de junio de 2020, en el sentido de que: "la pandemia de la COVID-19 no justifica ninguna restricción de los derechos fundamentales en el trabajo consagrados en las normas internacionales del trabajo, y el pleno respeto de esos derechos constituye una condición previa para entablar un diálogo social eficaz" (Observatorio de la OIT, 2020). Por su parte, y en este mismo sentido, aunque de manera atenuada, la Comisión Interamericana de Derechos Humanos (CIDH) y su Relatoría Especial sobre Derechos Económicos, Sociales, Culturales y Ambientales (REDESCA), sostienen que:

Cualquier medida de naturaleza restrictiva o regresiva con respecto a los DESCA, sea adoptada y aplicada de forma transparente, tras un cuidadoso análisis de las alternativas existentes. De adoptarse, dichas medidas deben estar justificadas desde un enfoque de 
derechos humanos con el debido análisis de impacto en los mismos, así como de la más eficiente utilización de los máximos recursos disponibles. (OEA, 2020)

\section{5. ¿HACIA UNA “NUEVA NORMALIDAD”?}

Una vez que se han implementado medidas emergentes para afrontar el inesperado brote del Covid-19 en el Ecuador, paulatina y progresivamente se está retornando a las actividades cotidianas, dejando atrás el confinamiento, en esta nueva etapa del distanciamiento social. En este escenario es fundamental considerar que la salud y seguridad ocupacional debe estar debidamente garantizada a todas las personas trabajadoras. De allí que el Comité de Derechos Económicos, Sociales y Culturales de las Naciones Unidas dentro de su Declaración sobre la pandemia de Covid-19 ha recomendado que los Estados adopten medidas para garantizar que se reduzcan al mínimo los riesgos de contagio. Hasta que se adopten tales medidas, sostiene el Comité, no se puede obligar a los trabajadores a trabajar y, a su vez, éstos deberían estar protegidos contra las sanciones disciplinarias o de otro tipo por negarse a trabajar sin la protección adecuada. Además, se recomienda que los Estados adopten medidas urgentes para proteger los empleos, las pensiones y otras prestaciones sociales de los trabajadores durante la pandemia, y así mitigar sus repercusiones económicas. (Naciones Unidas, 2020)

En este sentido, para efectos de la reactivación de las actividades laborales, se dispuso, mediante Decreto Ejecutivo 1074 (2020, Art.5), que el Ministerio de Trabajo realice los controles e inspecciones correspondientes a fin de que en el desarrollo de la jornada laboral se respeten tanto las medidas de bioseguridad necesarias, así como los derechos que deben garantizarse en toda relación laboral. Lo dicho se concretó en el AM MDT 093-2020, de 3 de mayo que fija las directrices para el retorno progresivo al trabajo. Entre tales directrices resulta conveniente destacar que la adopción de medidas de prevención corresponde al empleador. En este sentido, lo primero que debe hacer la parte empleadora que pretenda reiniciar las actividades 
laborales es observar de manera estricta la Guía aprobada por el Comité de Operaciones de Emergencia Nacional (COE). Será, por ende, responsabilidad del empleador: tomar las medidas de seguridad y salud en el trabajo, según corresponda a cada actividad. Además, prever la movilidad y logística a fin de que las personas trabajadoras arriben a sus lugares de trabajo, y una vez terminada su jornada laboral retornen a sus hogares, de manera segura, minimizando las posibilidades de contagio. Corresponde así mismo al empleador determinar los horarios, turnos, fechas y grupos de trabajadores para iniciar progresivamente las actividades en jornadas prolongadas o de recuperación. Entre las directrices consta también la necesidad de continuar con la modalidad de teletrabajo, siempre que sea posible, dando prioridad a los trabajadores en situación de vulnerabilidad. Se consideran tales a mujeres embarazadas, personas con discapacidad, mayores de sesenta y cinco años, o que adolezcan de enfermedades catastróficas o de alta complejidad.

Por su parte, la Dirección de seguridad, salud en el trabajo y gestión integral de riesgos del Ministerio de Trabajo expidió la guía de actuación para la prevención y control del Covid-19, durante la jornada presencial de los trabajadores. Esta establece los niveles de riesgos ocupacional (muy alto, alto, medio, bajo). Además, prescribe los protocolos para el manejo de trabajadores con casos sospechoso, probable y confirmado de Covid-19. (Ministerio de Trabajo, 2020)

Desde la declaratoria de emergencia sanitaria provocada por el Covid-19 hemos oído con gran insistencia la expresión "nueva normalidad", para referirse a la forma en que se desarrollará la vida cotidiana a partir del retorno progresivo a las actividades laborales y productivas, según la semaforización que para el efecto se ha establecido. Sin embrago, es preciso advertir que, si bien será una nueva realidad que implicará cambios en la forma de actuar, de hacer, de trabajar, de relacionarse, e inclusive de sentir, ello no será una situación permanente. En este sentido la filósofa Corina Dávalos (2020) sostiene que tanto el término de "normalidad" como el de "nueva" son absolutamente inadecuados para referirse 
a la situación en la que se vive luego de la declaratoria de la pandemia. El primero, por cuanto "normal" es lo que se ajusta a una norma. El segundo, porque alude al inicio de algo, que se experimenta por primera vez. Si se suman ambos términos resultará que "esta situación infrecuente e insólita es el inicio de algo que tomará posesión de nuestra cotidianeidad, sin que nadie lo perciba como inaceptable". Más aún si se considera que con ello se está generando un "marco mental" que solo deja ver lo que aparece en ese contorno, y así se ve la realidad tal como se la presenta. Al definir un marco mental, nos dice Dávalos (2020), se permite "a los gobiernos y medios que modifiquen nuestra manera de acercarnos a mirar y entender una realidad". Ese cambio de marco, según el lingüista George Lakoff, es un cambio social. Por lo tanto, advierte el peligro que supone la "nueva normalidad", ya que de manera insospechada con tal expresión se está formateando la percepción de lo que puede aprobarse como tolerable en el largo plazo. (citado por Dávalos, 2020)

En esta nueva etapa, a más de las medidas de bioseguridad que permitirán el retorno progresivo y seguro a las actividades laborales y productivas, será necesario implementar de manera urgente y prioritaria los mecanismos tendientes a una acelerada reactivación de la economía y de los empleos. Consecuentemente se espera, en el mundo del trabajo, como se expresa en la Ley de Apoyo Humanitario a propósito del contrato especial emergente, que se den nuevas inversiones o líneas de negocio, productos o servicios; ampliaciones o extensiones de los negocios, o modificación de los giros de los negocios; que se incremente la oferta de bienes y servicios por parte de personas naturales o jurídicas, nuevas o existentes. En definitiva, que se diversifiquen las fuentes de trabajo, que se generen nuevos emprendimientos, que se implementen suficientes estímulos para fortalecer las pequeñas y medianas empresas, a fin de que más personas puedan acceder a ingresos regulares y suficientes que les permitan superar los estragos de esta dura crisis y gozar de una vida digna. 


\section{REFLEXIONES FINALES}

La crisis provocada por el Covid-19 ha marcado un antes y un después en las relaciones laborales. Hasta antes de la declaratoria de la emergencia sanitaria, los trabajadores luchaban arduamente para no solo defender sus derechos, conquistados la gran mayoría de ellos a costo de sangre, sino para mejorarlos. Reclamaban cuando se cometían injusticias y sobre todo cuando se inobservaban las disposiciones del Código del Trabajo, los contratos, ya fueran individuales o colectivos, y fundamentalmente los principios del Derecho del Trabajo, cuya razón de ser es la protección de la parte débil de la relación laboral. Ahora los esfuerzos se limitan a los desesperados intentos por conservar la fuente de ingresos, resignando para ello, en muchos de los casos, sus derechos fundamentales.

La crisis también aceleró algunos cambios que se esperaban se fueran implementando de manera paulatina, en los diferentes órdenes de la vida social. Mencionaremos exclusivamente a los que corresponden a nuestro ámbito de análisis. Así, dichos cambios se presentaban desde hace algunos años como retos inaplazables del Derecho del Trabajo, producto de los avances de la ciencia y la tecnología y la cuarta revolución industrial para poner al mundo del trabajo acorde a las demandas de una acelerada e incontenible globalización y automatización. Nos referimos, por ejemplo, al impulso y aceptación de los medios telemáticos para la realización de las actividades laborales. Esto ha dado lugar inclusive al surgimiento de un nuevo derecho, el de la desconexión. Este nuevo derecho:

Se perfila como un aspecto clave para el trabajo propio de la era digital, donde las garantías de seguridad y salud sólo quedarán fortalecidas con la incorporación de nuevos límites, un cambio de mentalidad y una regulación específica. [...] lo que obliga a rediseñar la jornada laboral, a evaluar el desempeño en función de objetivos, y a aceptar la tendencia hacia una mayor autonomía del trabajador, puesto que los tradicionales parámetros de tiempo y lugar de trabajo y de descanso se difuminan. (Pérez, 2019) 
Así, "la inmersión en el mundo digital ha cambiado por completo la forma en la que se desarrolla la vida laboral (Pérez, 2019). No solo ello, sino que también obliga a asumir la realidad de que el trabajo va perdiendo cada vez más terreno debido a múltiples factores, que, al decir de Súpito (citado por Palomeque, 2011): "han contribuido a la erosión del poder de las conquistas obtenidas basadas en un trueque entre subordinación y estabilidad, también en el ámbito social". (p. 39) En esa tendencia es que las regulaciones emergentes que se expidieron para hacer frente a la crisis provocada por el Covid-19, inobservaron algunos principios esenciales del Derecho Laboral, concretamente, los principios de intangibilidad, progresividad, no regresividad y de irrenunciabilidad de los derechos de los trabajadores; del principio de la integridad y oportunidad de las remuneraciones; aunque dadas las circunstancias, muchas de tales medidas se consideraron legítimas.

La Organización Internacional del Trabajo, por su parte, sugiere algunos otros retos que serán necesarios asumir por parte de los gobiernos, para propiciar la creación de empleo, perdidos con motivo de la pandemia. Entre tales retos se encuentran: apoyar a los grupos vulnerables y los más afectados con el fin de lograr un mercado laboral más justo; y, fortalecer el diálogo social y el respeto de los derechos en el trabajo. Para ello será necesario remitirse a la Declaración del Centenario de la OIT sobre el futuro del trabajo adoptada en 2019, en virtud de la cual se establece un enfoque centrado en el ser humano para aumentar la inversión en la capacidad de las personas, las instituciones laborales y el empleo sostenible y decente en el futuro. Así también, ha fijado cuatro pilares fundamentales para abordar la crisis de la COVID-19 de conformidad con las normas internacionales del trabajo. Estos son: el estímulo de la economía y el empleo; el apoyo a las empresas, los empleos y los ingresos; la protección a los trabajadores en el lugar de trabajo; y, la búsqueda de soluciones mediante el diálogo social. (Observatorio OIT, 2020)

Por todo lo dicho, se hace inminente repensar el Derecho del Trabajo. Es necesario que ese nuevo derecho se encuentre a tono no solo con los efectos de la pandemia mundial y el consecuente requerimiento de una pronta 
reactivación económica, sino, sobre todo, acorde con un futuro que presenta muchos más desafíos, como son, la globalización, la tecnificación, y por ende las nuevas modalidades y condiciones de trabajo, fruto de la cuarta revolución industrial y el incremento del trabajo informal y autónomo. Millones de empleos en todo el mundo se perderán si no se da paso a la profunda transformación que exige la automatización de los diferentes procesos productivos y laborales. Es necesario, por lo tanto, que el Derecho Laboral acompase dichos cambios y se adapte a esas nuevas formas de organización del trabajo, sin perder su razón de ser, que es la protección de la parte trabajadora, pero no solo entendida de una relación laboral (dependiente y remunerada), sino de toda persona que se gane la vida trabajando. Esta redefinición o modernización del Derecho del Trabajo, "refundación institucional", al decir de Palomeque (2011), se viene debatiendo desde hace algunas décadas, como uno de los desafíos para entrar en el S XXI. (p. 40) Ello supone una revisión total del sistema normativo laboral y la flexibilización de los contenidos regulatorios, lo que "encierra en realidad transformaciones de envergadura y alcance desiguales, por lo que su aceptación [...] no debe ser necesariamente homogénea y funcional" (Palomeque, 2011, p. 40). En todo caso, concluye el mismo autor, que "la existencia de transformaciones institucionales [...] originadas por causas políticas y económicas es absolutamente consustancial al Derecho del Trabajo, de las que ha dado muestra permanente en sus diferentes expresiones históricas, y que derivan de modo esencial de la propia función social de respuesta de la norma laboral frente a la realidad social objeto de regulación". (Palomeque, 2011, p. 47)

Lastimosamente, pese a todo lo dicho, lejos de dar paso a una transformación profunda de este Derecho, de sus instituciones y regulaciones, lo que se ha realizado, particularmente en el Ecuador, han sido reformas desarticuladas y anacrónicas, y las últimas simplemente emergentes, a propósito del Covid-19 como era de esperar, manteniendo en el fondo un statu quo. Situación que no ha permitido esa urgente y constante adecuación del ordenamiento laboral a la realidad económica permanentemente cambiante. En definitiva, el 
Derecho del Trabajo está obligado a refundarse para adaptarse a las exigencias del trabajo del futuro y del futuro del trabajo, pero sin perder su razón de ser, como parte del Derecho Social, que es, "proteger y dignificar a las individuas social y económicamente débiles" (Stafforini, citado por Bosio 2019, p. 10). Se requiere, en definitiva, que el nuevo Derecho del Trabajo se proyecte al futuro del mundo del trabajo con una mirada diferente, innovadora y progresista para, centrado en el ser humano, ser más justo, equitativo e inclusivo. 


\section{REFERENCIAS BIBLIOGRÁFICAS}

Bosio, R. (2019). Lineamientos básicos del Derecho Social. T I. Córdoba-Argentina: Editorial Advócate.

Comisión Interamericana de Derechos Humanos (CIDH) y su Relatoría Especial sobre Derechos Económicos, Sociales, Culturales y Ambientales (REDESCA). (2020). Comunicado de prensa frente a la pandemia del COVID-19 (29-III-20). Recuperado de: http://www. oas.org/es/cidh/prensa/comunicados/2020/060.asp

Comité de Derechos Económicos, Sociales y Culturales de las Naciones Unidas (2020). Declaración sobre la pandemia de Covid-19. Recuperado de: http://hchr.org.mx/ images/doc_pub/Declaracin-sobre-la-pandemiade-Covid-19-y-los-derechos-econmicos-sociales-yculturales.pdf

Dávalos, C. (2020). La trampa de la nueva normalidad. Recuperado de: https://es.scribd.com/document/459867424/

Monesterolo Menciona, G. (2020). Régimen Jurídico Laboral de Empleados Privados y Obreros, 2da edición. Quito: Corporación de Estudios y Publicaciones y Centro de Publicaciones de la PUCE.

Organización Internacional del Trabajo (Observatorio) (2020). La Covid-19 y el mundo del trabajo, 5ta edición (30-V20). Recuperado de: https://www.ilo.org/wcmsp5/ groups/public/---dgreports/---dcomm/documents/ briefingnote/.

Páez Benalcázar, A. (2019). Principios del Derecho del Trabajo. Quito: Editora Jurídica Cevallos.

Palomeque López, C. (2011). Derecho del Trabajo e ideología, 7ma edición. España: Tecnos.

Pérez, A. I. (2019). La desconexión digital en España ¿un nuevo derecho laboral? Anuario jurídico y económico escurialense, ISSN 1133-3677, (52), pp. 101-124. 
Ramírez, J. (6 de marzo de 2020) Empleo en la cuarta revolución industrial. CE Noticias Financieras. Recuperado de: https://search.proquest.com/ docview/2373493481?accountid=13357

\section{Base Legal}

Asamblea Nacional del Ecuador. Ley de Apoyo Humanitario para Combatir la Crisis Sanitaria Derivada del Covid-19. [Ley]. (16 de junio de 2020). R. O. 225 de 16 de junio de 2020.

Congreso Nacional del Ecuador. Código del Trabajo. [Codificación 17 de 2005]. (16 de diciembre de 2005). RO. 167 de 16 de diciembre de 2005.

Constitución de la República del Ecuador [Const.]. (2008). 2da Ed. CEP.

Ministerio del Trabajo. (2020). Acuerdo Ministerial MDT 0812020-, reforma el Acuerdo MDT 135-2017, referente al instructivo para el cumplimiento de las obligaciones de los empleadores públicos y privados (10-IV-20).

Ministerio del Trabajo. (2020). Acuerdo Ministerial MDT 076-2020, teletrabajo emergente (MDT 12-III-20).

Ministerio del Trabajo. (2020). Acuerdo Ministerial MDT 0772020-, directrices para la aplicación de la reducción, modificación o suspensión emergente de la jornada laboral durante la declaratoria de emergencia sanitaria (MDT 15-III-20).

Ministerio del Trabajo. (2020). Acuerdo Ministerial MDT 079-2020, reforma al instructivo de cumplimiento de obligaciones de los empleadores públicos y privados (MDT 24-III-20).

Ministerio del Trabajo. (2020). Acuerdo Ministerial MDT 0802020, reforma al AM 077-2020 (28-III-20). 
Ministerio del Trabajo. (2020). Acuerdo Ministerial MDT 093-2020, directrices para la reactivación económica a través del retorno progresivo al trabajo del sector privado (03-V-20).

Ministerio del Trabajo. (2020). Acuerdo Ministerial MDT 1322020-, directrices para el registro de las modalidades y acuerdos laborales establecidos en el capítulo II de la Ley de Apoyo Humanitario (15-VII-20)

Ministerio del Trabajo. (2020). Acuerdo Ministerial MDT 1332020-, directrices para la aplicación de la reducción emergente de la jornada de trabajo establecida en la Ley de Apoyo Humanitario (15-VII-20).

Presidente de la República. (2020). Decreto Ejecutivo 1074 (RO-S 225, 16-VI-20).

\section{RESOLUCIONES}

Ministerio de Trabajo. (2020). Resolución 2020-23 que reforma la Resolución 2020-22, sobre la calificación del Covid-19 como enfermedad profesional (MDT, 29-IV-20).

Recibido: $30 / 05 / 2020$

Aprobado: 30/07/2020

Graciela Monesterolo Lencioni: Profesora titular principal PUCE. Doctora en Jurisprudencia y Magíster por la Pontificia Universidad Católica del Ecuador.

Correo: GAMONESTEROLO@puce.edu.ec

Ciudad: Quito

País: Ecuador 
